\title{
Evaluation of Raman spectroscopy in comparison to commonly performed dengue diagnostic tests
}

Saranjam Khan

Rahat Ullah

Muhammad Khurram

Hina Ali

Arshad Mahmood

Ajmal Khan

Mushtaq Ahmed 


\title{
Evaluation of Raman spectroscopy in comparison to commonly performed dengue diagnostic tests
}

\author{
Saranjam Khan, ${ }^{\mathrm{a}, \boldsymbol{}}$ Rahat Ullah, ${ }^{\mathrm{a}}$ Muhammad Khurram, ${ }^{\mathrm{b}}$ Hina Ali, ${ }^{\mathrm{a}}$ Arshad Mahmood,, ${ }^{\mathrm{a}}$ Ajmal Khan,, ${ }^{\mathrm{c}}$ and \\ Mushtaq Ahmed ${ }^{\text {a }}$ \\ ${ }^{a}$ National Institute for Lasers and Optronics, Agri-Biophtonics Division, Nilore, Islamabad 45650, Pakistan \\ ${ }^{\mathrm{b}}$ Rawalpindi Medical College, Department of Medicine, Rawalpindi 46000, Pakistan \\ ${ }^{\circ}$ COMSATS Institute of Information Technology, Department of Chemistry, Abbottabad 22060, Pakistan
}

\begin{abstract}
This study demonstrates the evaluation of Raman spectroscopy as a rapid diagnostic test in comparison to commonly performed tests for an accurate detection of dengue fever in human blood sera. Blood samples of 104 suspected dengue patients collected from Holy Family Hospital, Rawalpindi, Pakistan, have been used in this study. Out of 104 samples, $52(50 \%)$ were positive based on immunoglobulin $\mathrm{G}(\mathrm{IgG})$, whereas $54(52 \%)$ were positive based on immunoglobulin M (IgM) antibody tests. For the determination of the diagnostic capabilities of Raman spectroscopy, accuracy, sensitivity, specificity and false positive rate have been calculated in comparison to normally performed IgM and IgG captured enzyme-linked immunosorbent assay tests. Accuracy, precision, specificity, and sensitivity for Raman spectroscopy in comparison to IgM were found to be $66 \%, 70 \%, 72 \%$, and $61 \%$, whereas based on IgG they were $47 \%, 46 \%, 52 \%$, and $43 \%$, respectively. $\odot 2016$ Society of Photo-Optical Instrumentation Engineers (SPIE) [DOI: 10.1117/1.JBO.21.9.095005]
\end{abstract}

Keywords: Raman spectroscopy; specificity; sensitivity; positive predictive value; negative predictive value; immunoglobulin M; immunoglobulin G.

Paper 160299RR received May 15, 2016; accepted for publication Aug. 31, 2016; published online Sep. 26, 2016.

\section{Introduction}

Dengue infection is mosquito-borne viral disease resulting from the bite of a mosquito infected with the dengue virus. Most common symptoms include sudden high fever, severe headache, body rashes, muscular pain, nausea, and vomiting. The symptoms usually appear 4 to 7 days after the bite of the infected mosquito and last for the 10 days. Currently, there is no vaccine nor medicine to treat dengue infected patients. The doctors rely only on supportive medicine and maintaining body fluids. The most critical thing in controlling epidemics and casualties is the early and accurate diagnosis of the disease. According to WHO estimation, dengue virus causes about 50 to 100 million infections annually worldwide, and the disease is now endemic in more than 100 countries. $^{1-4}$

The diagnostics depend on case study, case management, surveillance, and outbreak investigations. The optimum time for diagnosis of dengue fever is from onset of disease to 10 days postinfection. Different types of laboratory tests are usually performed at a hospital for the confirmation of this disease. These tests are based on direct virus detection or detecting antibodies in the blood or serum of the suspected patient. Virus detectionbased methods are usually effective in the early 5 days; however, the cost, expertise, and time required until results are available (1 week) limit its use in screening the suspected patients suffering from fever. ${ }^{5,6}$ Moreover, in countries where the population is large with limited facilities, it is not possible to perform expensive diagnostic tests for screening purposes at a mass scale. Thus, there is dire need for the development of a test which is quick, economical, and user-friendly.

${ }^{\star}$ Address all correspondence to: Saranjam Khan, E-mail: k.saranjam @yahoo com
Medically, a test with high sensitivity and specificity is usually preferred. Normally, these types of laboratory tests are available but are more complex, laborious, and expensive. In Pakistan, the nonstructural protein 1 (NS1) test is initially performed in the hospital for all patients having symptoms of dengue fever. ${ }^{7-9}$ The test has high sensitivity but unfortunately a very low specificity. Another commonly performed test that can separate primary and secondary infections is enzyme-linked immunosorbent assay (ELISA), which is less expensive and easier to perform as compared to virus isolation and nucleic acidbased detection. Before performing an ELISA test, a diagnostic test with a high specificity is required to accurately point out the patients with the disease.

In the last decade, Raman spectroscopy has gained wide popularity in the field of biomedical research and medical diagnosis. ${ }^{10,11}$ Raman spectroscopy is inelastic scattering of light photons by the molecular vibration inside the sample. As a result, the scattered photons are emitted with the different wavelength. This difference in frequency between the two is termed the Raman shift. The Raman spectrum is basically the plot of intensity as a function of wave number (Raman shift). There are different bands in the Raman spectrum. Each band represents a specific molecular bond inside the sample. ${ }^{12,13}$ In different types of diseases, initial changes started at the molecular level. Raman spectroscopy has the ability to monitor these changes, which is necessary for an early diagnosis. This article is based on Raman spectroscopy evaluation for early diagnosis of dengue fever in comparison to immunoglobulin $\mathrm{M}$ (IgM) and immunoglobulin $\mathrm{G}$ ( $\mathrm{IgG}$ ) captured ELISA. For the evaluation of Raman spectroscopy, different statistical terms such as accuracy, sensitivity, specificity, and false positive rate have been calculated. Raman spectroscopy is fast, cost effective, and also does

$1083-3668 / 2016 / \$ 25.00$ @ 2016 SPIE 
not require a complicated sample preparation procedure. This technique is still in the research phase and required a lot of different types of samples. Once it is established it will be quite helpful in the field of medical diagnosis and biomedical research.

\section{Materials and Methods}

\subsection{Sample Collection and Preparation}

In total, 104 samples of different ages and genders have been used for the current analysis. All these samples have been acquired directly from Holy Family Hospital, Rawalpindi, Pakistan, at different days in the months of September and October 2015. These samples were initially declared dengue positive on the basis of common symptoms and physical condition of the patient. A quantity of about $6 \mathrm{ml}$ blood was obtained from each patient by using a sterile syringe prior to any medication. The samples were collected in clot activator tubes (HebeiXinle, Sci\&Tech Co. Ltd., China). A $3 \mathrm{ml}$ portion of each sample has been used for IgG and IgM captured ELISA, whereas the remaining $3 \mathrm{ml}$ has been used for Raman spectroscopy. All samples have been centrifuged at $3500 \mathrm{rpm}$ for $10 \mathrm{~min}$ using a Hettich Centrifuge D-7200 for the serum extraction. The extracted sera have been aliquoted in different tubes and stored at $-16^{\circ} \mathrm{C}$ till further use in Raman spectroscopy. The sample preparation and storage procedures are same as mentioned in our previous article. ${ }^{14,15}$ The overall experimental procedure has been carried out after obtaining written approval from the Research and Ethical Committee of Rawalpindi Medical College and Allied Hospital Rawalpindi. The standard safety rules $^{16}$ have been followed during all experimental procedures.

\subsection{Acquiring Raman Spectra}

A sample quantity of about 15 to $20 \mu \mathrm{l}$ of every sample was placed on the glass slide and dried for about $2 \mathrm{~h}$ at room temperature. A Raman spectrometer ( $\mu$ Ramboss DONGWOO OPRTON, South Korea) with a spectral resolution of $4 \mathrm{~cm}^{-1}$ was used for recording Raman spectra. A laser diode emitting continuous laser beam at $532 \mathrm{~nm}$ has been used for the excitation. A microscope objective having a magnification of $100 \times$ has been used both for focusing light on the sample and collection of Raman scattered light. A $15 \mathrm{~s}$ acquisition time has been used for recording each spectral data. The spectral range from 600 to $1700 \mathrm{~cm}^{-1}$, as it contained the most useful information, has been used for recording Raman spectra for all samples. A sketch of the experimental setup is the same as previously published. ${ }^{17}$

\section{Results}

\subsection{Sample Analysis}

Biological samples normally contain large numbers of biomolecules. Each molecule gives rise or contributes to a specific peak in the spectrum. The noise from different types of background sources also exists in the Raman spectra. Most of the time the background noise is so strong that it dominates the weak Raman signal as only 1 out of $10^{6}$ get Raman scattered. The biomolecules existing in the sample are considered as a strong source of the fluorescence. Before making a spectral analysis, it is necessary to first remove the entire background signal in order to get a noise-free spectrum. For this purpose, a code has been developed in the MATLAB environment. Initially, all 104 samples have been denoised. All these denoised spectra were then smoothed using a Savitzky-Golay filter with five points taken at a time and fitting a third-order polynomial. ${ }^{15}$

\subsection{Raman Spectral Analysis}

The Raman spectra of normal as well as infected human blood sera are shown in Fig. 1. For the purpose of demonstration, the spectra of normal blood sera are shown in dotted green line, whereas the spectra of infected sera are shown in magenta color. The Raman spectra of the normal blood serum contain its three most prominent peaks at 1003, 1156, and $1516 \mathrm{~cm}^{-1} \cdot{ }^{15,17,18}$ The Raman peak at $1003 \mathrm{~cm}^{-1}$ has been assigned to the symmetric ring breathing mode of phenylalanine and carotene, whereas the peaks at 1156 and $1516 \mathrm{~cm}^{-1}$ have been assigned to $\beta$-carotene. ${ }^{14,17-19}$ These three peaks are always reproducible in the serum of healthy individuals. In infected samples, these three peaks get suppressed and new peaks appear at different frequencies. All those samples which do not contain the above stated peaks are considered as abnormal and vice versa. In the Raman spectra of dengue infected serum, new peaks appeared at $750,850,1450$, and $1660 \mathrm{~cm}^{-1}$. The assignments of all additional peaks appearing in cases of dengue infection are previously published. ${ }^{15}$ Among the 104 samples used in this study, 57 are clearly dengue negative (normal), whereas 47 are dengue positive based on the Raman analysis. These results were then compared with the physician results (truth table), which were kept hidden initially.

Different types of statistical terms such as accuracy, sensitivity, and specificity are calculated by using a statistical algorithm. Statistical methods generate a confusion matrix which consists of four elements, namely, true positive, true negative, false positive, and false negative. These four elements were later used in the calculation of all statistical terms used for the evaluation of different types of diagnostic tests. In this study, these four elements have been calculated directly from the comparison of Raman findings with the ELISA (IgG and $\operatorname{IgM}$ ) results obtained from hospital. As mentioned before these results were initially coded. The Raman findings were compared separately with the IgM and IgG results of the physician. In this case, the true positive is the one which is positive according to the Raman as well as ELISA findings, whereas true negatives are those which are negative according to both. In contrast, false positives are those which are positive on the Raman basis but negative according to ELISA, and false negative results are those which are negative according to Raman but negative on the ELISA basis. Using these four elements, all the statistical terms have been calculated.

\section{Discussion}

As mentioned before, the basic aim of the ongoing study is the evaluation of Raman spectroscopy as a screening test for the detection of dengue fever. Different types of screening tests are normally performed at different hospitals. Indeed, a screening test is required that is simple, fast, and cost effective. A test with high sensitivity and specificity is usually preferred. Accuracy, specificity, and sensitivity are the most widely used statistical measures for the evaluation of the clinical tests. Sensitivity is a test's ability to correctly point out the presence of a disease in a person who has in fact that disease. Specificity is the test's ability to clearly indicate the absence of the disease when the patient is really disease free. Similarly, accuracy is the 


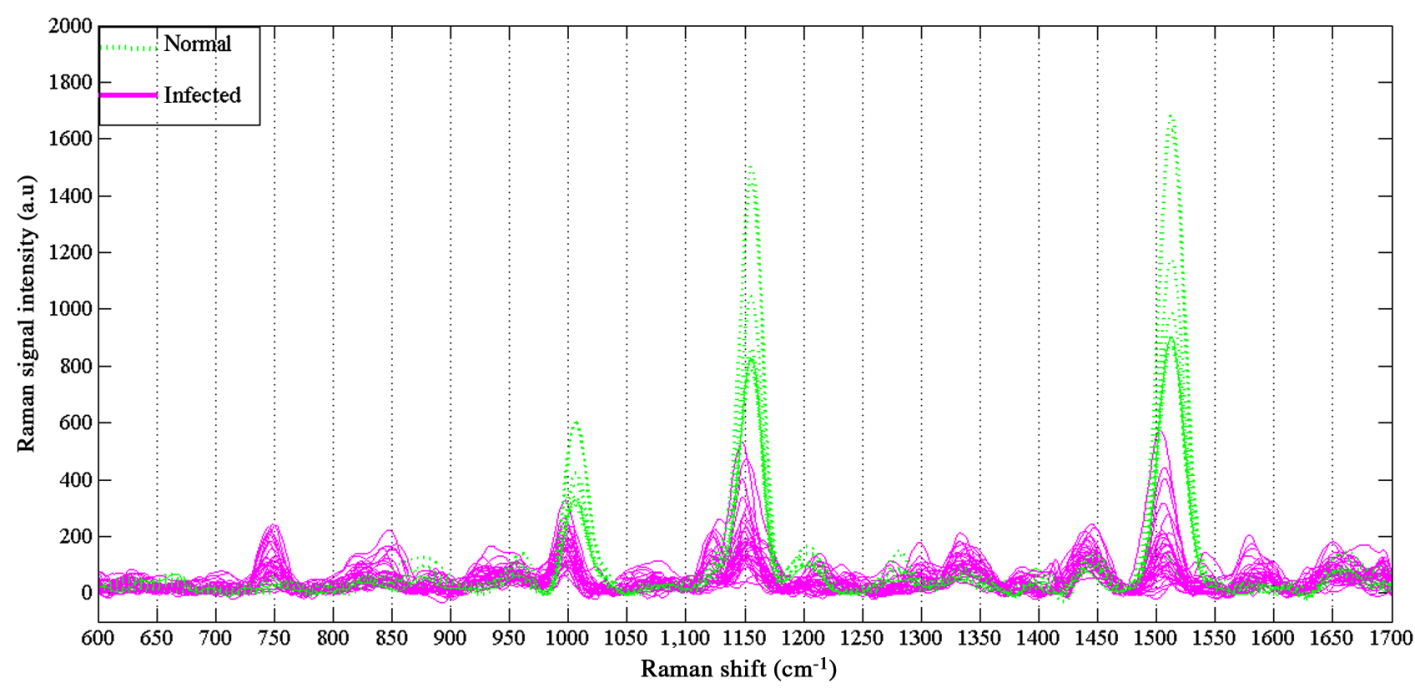

Fig. 1 Raman spectra of normal healthy volunteer (dotted green line) and dengue infected patient (magenta).

ratio of correctly predicted results to the total number of predictions.

In total, 104 samples have been analyzed in this study. Three different tests, namely, ELISA (IgM, IgG), and Raman have been performed for all the above samples. Normally, dengue virus isolation or detection of RNA is considered as the gold standard test for dengue diagnosis. These tests are expensive, time consuming, and unable to differentiate between primary and secondary infections. Additionally, a contamination may lead to a chance of getting false positive results. These are the basic limitations regarding viral isolation or detection. Serologically based tests focusing on dengue NS1 antigen detection, dengue specific IgM and IgG, are extensively used for dengue diagnosis as these are less expensive and faster, and differentiate between primary and secondary infections. At our center, the SD Dengue Capture ELISA Kit for dengue IgM and IgG detection is used for this purpose. For IgM, its sensitivity is $96.4 \%$, and its specificity is $98.9 \%$. For IgG, its sensitivity is $98.8 \%$, and its specificity is $99.2 \%$. All these tests are used based on Dengue Expert Advisory Group (DEAG) guidelines for diagnosing dengue infection. ${ }^{20,21}$ Since the main theme of this study is the evaluation of Raman spectroscopy as a diagnostic tool with reference to dengue specific $\operatorname{IgM}$ and $\mathrm{IgG}$. Therefore, for this particular study, the results of the ELISA test have been considered as the gold standard as it differentiates between primary and secondary infections. The ELISA test predicts both $\operatorname{IgM}$ and $\operatorname{IgG}$ antibodies. The response of antibodies to infection varies depending on the immune system of the infected individual. In a patient with the primary infection, IgM antibodies are produced first and are detectable in $80 \%$ of the patients by day 5 of the onset, whereas IgG antibodies are normally detectable after day 10 and exist probably for the life time. In contrast, in patients with the secondary infection, IgM produces in the same time period at low titres, but IgG exists from the previous infection, and the titres increase rapidly. ${ }^{5,9}$ Accuracy, sensitivity, specificity, positive predicative value (precision), and negative predicative values are calculated for Raman spectroscopy in comparisons to ELISA based on IgM and IgG separately as given in Table 1 .

Accuracy, precision, specificity, and sensitivity of the Raman process in comparisons to $\operatorname{IgM}$ were found to be $66 \%, 70 \%$,
$72 \%$, and $61 \%$, whereas for IgG-based analysis they were $48 \%, 48 \%, 52 \%$, and $44 \%$, respectively. The most obvious reason for the low sensitivity and specificity of IgG-based results is that all samples had been collected at the time of admission, and initially there is higher probability of $\operatorname{IgM}$ rather than $\operatorname{IgG}$, which is detectable after 10 to 15 days. ${ }^{9,22}$ The reason for the overall lower sensitivity of Raman analysis is most likely more false negative results. As mentioned before, the Raman spectrum of the normal blood sera samples shows three intense peaks at 1003,1156 , and $1516 \mathrm{~cm}^{-1}$. A situation where only these three peaks exist with no additional peaks found is certainly dengue negative. The negative results of Raman spectroscopy are more reliable for two reasons, first due to the presence of only three dominant Raman lines at the above-mentioned Raman shift, and second because of its reproducibility. In our case, there are 21 samples (20\%) for which Raman contradicts with the IgM values of the ELISA findings. According to the

Table 1 Comparison of Raman findings and ELISA results.

\begin{tabular}{lcc}
\hline Type of analysis & $\begin{array}{c}\text { Raman } \\
\text { spectroscopy } \\
\text { versus IgM }\end{array}$ & $\begin{array}{c}\text { Raman } \\
\text { spectroscopy } \\
\text { versus IgG }\end{array}$ \\
\hline True positive & 33 & 23 \\
True negative & 36 & 27 \\
False positive & 14 & 25 \\
False negative & 21 & 29 \\
Accuracy & $66 \%$ & $48 \%$ \\
Specificity & $72 \%$ & $52 \%$ \\
Sensitivity & $61 \%$ & $44 \%$ \\
$\begin{array}{l}\text { Precision (positive } \\
\text { predicative value) }\end{array}$ & $70 \%$ & $48 \%$ \\
Negative predicative & & \\
value & $63 \%$ & $48 \%$ \\
\hline
\end{tabular}


Raman, these 21 samples are dengue negative, but ELISA predicted as positive for these samples.

High sensitivity is no doubt very important in screening tests for the identification of disease in the population. But most often a test with high sensitivity and low specificity declares many disease-free persons to have a disease. Such tests are quite good for the region where the population is smaller and the resources are sufficient. In a country where the population is greater and resources are limited, a test with high specificity is more favorable than a test with high sensitivity.

\section{Conclusion}

This article presents the evaluation of Raman spectroscopy as a screening test for the diagnosis of dengue virus infection in human blood sera. Raman spectroscopy has comparatively better specificity as well as sensitivity and a very low false positive rate in comparison to IgM than IgG. IgM antibodies are produced first; therefore, the IgM-based test is more effective when performed in the first week of infection. So, there is also a possibility to use Raman spectroscopy in combination with other screening test such as NS1 during initial screening for increasing the diagnostics efficiency. The results obtained are quite promising and interesting. The research work in our laboratory is still in progress, striving for increasing sensitivity as well as specificity through Raman spectroscopy.

\section{Acknowledgments}

We are thankful to Staff Nurse Uzma Shazia, Dengue Ward Holy Family Hospital Rawalpind; Mrs. Fatima Batool; and Muhammad Irfan, scientific assistant, Agri-Biophotonics Division at the National Institute for Lasers and Optronics, for assisting the present research work. The authors have no relevant financial interests in this article and no other potential conflicts of interest to disclose.

\section{References}

1. World Health Organization Report, "Dengue and severe dengue," WHO (2015).

2. C. C. Carlos et al., "Comparison of clinical features and hematologic abnormalities between dengue fever and dengue hemorrhagic fever among children in the Philippines," Am. J. Trop. Med. Hyg. 73(2), 435-440 (2005).

3. D. Guha-Sapir and B. Schimmer, "Dengue fever: new paradigms for a changing epidemiology," Emerg. Themes Epidemiol. 2(1), 1 (2005).

4. J. Whitehorn and J. Farrar, "Dengue," Br. Med. Bull. 95(1), 161-173 (2010).

5. World Health Organization Report, "World Health Organization guideline for diagnosis, treatment, prevention and control," 2009 (18 February 2016).
6. V. Wiwanitkit, "Dengue fever: diagnosis and treatment," Expert Rev, Anti-Infect. Ther. 8(7), 841-845 (2010).

7. R. Parikh et al., "Understanding and using sensitivity, specificity and predictive values," Indian J. Ophthalmol. 56(1), 45-50 (2008).

8. F. M. Kassim et al., "Use of dengue NS1 antigen for early diagnosis of dengue virus infection," Southeast Asian J. Trop. Med. Public Health 42(3), 562-569 (2011).

9. R. W. Peeling et al., "Evaluation of diagnostic tests: dengue," Nat. Rev. Microbiol. 8(12 Suppl.), S30-S38 (2010).

10. P. Crow et al., "The use of Raman spectroscopy to identify and characterize transitional cell carcinoma in vitro," BJU Int. 93(9), 1232-1236 (2004).

11. C. A. Owen et al., "Progress in Raman spectroscopy in the fields of tissue engineering, diagnostics and toxicological testing," J. Mater. Sci. Mater. Med. 17(11), 1019-1023 (2006).

12. J. J. Laserna, "An introduction to Raman spectroscopy: introduction and basic principles," Raman/Infrared Spectroscopy (2014).

13. A. Lorincz et al., "Raman spectroscopy for neoplastic tissue differentiation: a pilot study," J. Pediatr. Surg. 39(6), 953-956 (2004).

14. M. Saleem et al., "Optical diagnosis of dengue virus infection in human blood serum using Raman spectroscopy," Laser Phys. Lett. 10(3), 035602 (2013).

15. S. Khan et al., "Raman spectroscopic analysis of dengue virus infection in human blood sera," Opt. Int. J. Light Electron Opt. 127(4), 20862088 (2016).

16. US Department of Health and Human Services, Biosafety in Microbiological and Biomedical Laboratories, 5th ed., U.S. Department of Health and Human Services (2009).

17. S. Khan et al., "Analysis of dengue infection based on Raman spectroscopy support vector machine (SVM)," Biomed. Opt. Express 7(6), 2249-2256 (2016).

18. Z. Movasaghi, S. Rehman, and I. U. Rehman, "Raman spectroscopy of biological tissues," Appl. Spectrosc. Rev. 42(5), 493-541 (2007).

19. C. Painter and J. L. Koenig, "Raman spectroscopic study of the structure of antibodies," Biopolymers 14(3), 457-468 (1975).

20. M. Khurram et al., "Dengue hemorrhagic fever: comparison of patients with primary and secondary infections," J. Infect. Public Health 7(6), 489-495 (2014).

21. T. K. Butt, M. Ali, and F. Masud, "Dengue GCP Guidelines.pdf," 2012, http://sims.edu.pk/Dengue/DengueGCPGUIDELINES.pdf (4 May 2016).

22. A. Sa-Ngasang et al., "Specific IgM and IgG responses in primary and secondary dengue virus infections determined by enzyme-linked immunosorbent assay," Epidemiol. Infect. 134(4), 820-825 (2006).

Saranjam Khan received his MSc degree in physics and his MS degree in nuclear engineering from the University of Peshawar, Pakistan, in 1999 and Pakistan Institute of Engineering and Applied Science in 2002. He received his $\mathrm{PhD}$ in applied optics from the Division of Biomedical Physics, Innsbruck Medical University, Innsbruck, Austria, in 2011. He is a principal scientist at the National Institute for Lasers and Optronics. He is the author of nine journal papers. His current research interests include Raman spectroscopy, fluorescence spectroscopy, and microscopy.

Biographies for the other authors are not available. 